DESY 09-055

TUM-HEP 720/09

June 2009

\title{
Probing Gravitino Dark Matter with PAMELA and Fermi
}

\author{
Wilfried Buchmüller ${ }^{a}$, Alejandro Ibarra ${ }^{b}$, Tetsuo Shindou ${ }^{c}$, \\ Fumihiro Takayama ${ }^{a}$ and David $\operatorname{Tran}^{b}$ \\ ${ }^{a}$ Deutsches Elektronen-Synchrotron DESY, 22607 Hamburg, Germany \\ ${ }^{b}$ Physik-Department T30d, Technische Universität München, 85748 Garching, Germany \\ ${ }^{c}$ Kogakuin University, 1638677 Tokyo, Japan
}

\begin{abstract}
We analyse the cosmic-ray signatures of decaying gravitino dark matter in a modelindependent way based on an operator analysis. Thermal leptogenesis and universal boundary conditions at the GUT scale restrict the gravitino mass to be below $600 \mathrm{GeV}$. Electron and positron fluxes from gravitino decays, together with the standard GALPROP background, cannot explain both, the PAMELA positron fraction and the electron + positron flux recently measured by Fermi LAT. For gravitino dark matter, the observed fluxes require astrophysical sources. The measured antiproton flux allows for a sizable contribution of decaying gravitinos to the gamma-ray spectrum, in particular a line at an energy below $300 \mathrm{GeV}$. Future measurements of the gamma-ray flux will provide important constraints on possible signatures of decaying gravitino dark matter at the LHC.
\end{abstract}




\section{Introduction}

An unequivocal prediction of locally supersymmetric extensions of the Standard Model is the gravitino, the gauge fermion of supergravity [1]. Depending on the mechanism of supersymmetry breaking, it can be the lightest superparticle, which makes it a natural dark matter candidate [2]. In connection with thermal leptogenesis [3], gravitino dark matter has been discussed as an alternative [4] to the standard WIMP scenario [5].

In a class of models with small $R$-parity and lepton number breaking the gravitino is no longer stable, but its decays into Standard Model (SM) particles are doubly suppressed by the Planck mass and the small $R$-parity breaking parameter. Hence, its lifetime can exceed the age of the Universe by many orders of magnitude, and the gravitino remains a viable dark matter candidate [6]. Recently, it has been shown that such models yield a consistent cosmology incorporating nucleosynthesis, leptogenesis and gravitino dark matter [7].

Small $R$-parity breaking can arise from spontaneous B-L breaking [7] or from left-right symmetry breaking [8]. Alternatively, explicit $R$-parity violating couplings of heavy Majorana neutrinos can lead to suppressed $R$-parity breaking interactions in the low energy effective theory via the seesaw mechanism [9]. In the simplest supergravity models with universal gaugino masses at the Grand Unification (GUT) scale, thermal leptogenesis implies an upper bound of $600 \mathrm{GeV}$ on the gravitino mass [10]. Relaxing the boundary conditions at the GUT scale, gravitino masses up to $1.4 \mathrm{TeV}$ are possible [11].

Gravitino decays may lead to characteristic signatures in high-energy cosmic rays. The produced flux of gamma rays $[6,7,12-15]$ and positrons $[15,16]$ has been found to potentially account for the extragalactic component of the excess in the EGRET [17] and HEAT [18] data, respectively. Furthermore, a neutrino flux from gravitino decays is predicted [19] as well as a possibly observable antideuteron flux [20].

Recently, a steep rise in the cosmic-ray positron fraction above $10 \mathrm{GeV}$ has been discovered by the PAMELA collaboration [21] whereas the observed antiproton-to-proton ratio [22] is consistent with previous measurements of the antiproton flux by BESS [23], IMAX [24] and WiZard/CAPRICE [25]. A possible explanation of this exotic positron source is annihilating or decaying dark matter [26], including decaying gravitinos [27, 28]. Equally important are the recent measurements of the total electron + positron flux by ATIC [29], H.E.S.S. [30] and Fermi LAT [31].

In this paper we analyse the cosmic-ray signatures of decaying gravitino dark matter in a model-independent way based on an operator analysis. Consistency with the observed antiproton flux yields a lower bound on the gravitino lifetime. As we shall see, this determines an upper bound on the continuous gamma-ray spectrum. Following a previous analysis of supergravity models and leptogensis, we only consider gravitino masses below $600 \mathrm{GeV}$. Hence, gravitino decays cannot be the cause of the anomaly observed by the ATIC [29] and Fermi LAT [31] collaborations. An interpretation of the PAMELA positron anomaly as the result of gravitino dark matter decay, on the other hand, requires gravitino masses above $200 \mathrm{GeV}$.

This paper is organised as follows. In Section 2 we present a general operator analysis of gravitino decays and study the implications for the different branching ratios. In 
particular we discuss the strength of the predicted monochromatic line in the gamma-ray spectrum. Section 3 deals with the electron, positron and antiproton flux from gravitino decays and the implications for the gravitino lifetime. The results for the gamma-ray spectrum are discussed in Section 4, followed by our conclusions in Section 5.

\section{Gravitino decays}

$R$-parity violating gravitino decays are conveniently described in terms of effective operators. The spinor of a massive gravitino (cf. [32]) satisfies the Dirac equation

$$
\left(i \gamma^{\mu} \partial_{\mu}-m_{3 / 2}\right) \psi_{\nu}=0
$$

together with the constraints

$$
\gamma^{\mu} \psi_{\mu}=0, \quad \partial^{\mu} \psi_{\mu}=0
$$

The mass scales multiplying the non-renormalizable operators are inverse powers of the Planck mass $M_{\mathrm{Pl}}$ and the supersymmetry breaking gravitino mass $m_{3 / 2}$. This assumes for the masses $m_{\mathrm{SM}}$ of Standard Model particles, the gravitino mass and the masses $m_{\text {soft }}$ of other superparticles the hierarchy $m_{\text {SM }}^{2} \ll m_{3 / 2}^{2} \ll m_{\text {soft }}^{2}$.

Using Eqs. (2.1) and (2.2) one easily verifies that the dimension-5 and dimension-6 operators for $R$-parity violating couplings of the gravitino to Standard Model particles are given by

$$
\mathcal{L}_{\mathrm{eff}}=\frac{i \kappa}{\sqrt{2} M_{\mathrm{Pl}}}\left\{\bar{l} \gamma^{\lambda} \gamma^{\nu} D_{\nu} \phi \psi_{\lambda}+\frac{i}{2} \bar{l} \gamma^{\lambda}\left(\xi_{1} g^{\prime} Y B_{\mu \nu}+\xi_{2} g W_{\mu \nu}\right) \sigma^{\mu \nu} \phi \psi_{\lambda}\right\}+\text { h.c. }
$$

where typically $\xi_{1.2}=\mathcal{O}\left(1 / m_{3 / 2}\right)$. Note, however, that in general $\kappa$ and the product $\kappa \xi_{1,2}$ are independent parameters. For simplicity, we have suppressed the flavour indices of $\kappa$, $l$ and $\xi_{1,2}$. The covariant derivative involves the $\mathrm{U}(1)$ and $\mathrm{SU}(2)$ gauge fields $B_{\mu}$ and $W_{\mu}$, respectively,

$$
D_{\mu}=\partial_{\mu}+i g^{\prime} Y B_{\mu}+i g W_{\mu}, \quad Y[\phi]=-\frac{1}{2}, \quad W_{\mu}=\frac{1}{2} \sigma^{I} W_{\mu}^{I},
$$

with the corresponding field strengths

$$
B_{\mu \nu}=\partial_{\mu} B_{\nu}-\partial_{\nu} B_{\mu}, \quad W_{\mu \nu}=\partial_{\mu} W_{\nu}-\partial_{\nu} W_{\mu}+i\left[W_{\mu}, W_{\nu}\right] .
$$

In the unitary gauge, the Higgs and lepton doublets read

$$
\phi=\left(\begin{array}{c}
v+\frac{1}{\sqrt{2}} h \\
0
\end{array}\right), \quad l=\left(\begin{array}{l}
\nu \\
e
\end{array}\right)
$$

where $h, \nu$ and $e$ denote Higgs boson, neutrino and charged lepton, respectively. 
From Eq. (2.3) one easily obtains the couplings of leptons and gravitino to Higgs and gauge bosons, which are responsible for the two-body gravitino decays,

$$
\begin{aligned}
\mathcal{L}_{3} \supset \frac{i \kappa}{\sqrt{2} M_{\mathrm{Pl}}}\{ & \left(\partial_{\mu} h+i m_{Z} Z_{\mu}\right) \bar{\nu} \gamma^{\nu} \gamma^{\mu} \psi_{\nu}+i \sqrt{2} m_{W} W_{\mu}^{-} \bar{e} \gamma^{\nu} \gamma^{\mu} \psi_{\nu} \\
& +i m_{Z}\left(\xi_{Z} \partial_{\mu} Z_{\nu}+\xi_{\gamma} \partial_{\mu} A_{\nu}\right) \bar{\nu} \gamma^{\lambda} \sigma^{\mu \nu} \psi_{\lambda} \\
& \left.+i \sqrt{2} m_{W} \xi_{W} \partial_{\mu} W_{\nu}^{-} \bar{e} \gamma^{\lambda} \sigma^{\mu \nu} \psi_{\lambda}\right\}+ \text { h.c. }
\end{aligned}
$$

with

$$
\begin{aligned}
& \xi_{Z}=\sin ^{2} \theta_{\mathrm{W}} \xi_{1}+\cos ^{2} \theta_{\mathrm{W}} \xi_{2}, \quad \xi_{W}=\xi_{2}, \\
& \xi_{\gamma}=\sin \theta_{\mathrm{W}} \cos \theta_{\mathrm{W}}\left(\xi_{2}-\xi_{1}\right), \quad \sin \theta_{\mathrm{W}}=\frac{g^{\prime}}{\sqrt{g^{\prime 2}+g^{2}}} .
\end{aligned}
$$

Note that the gauge boson couplings satisfy the relation

$$
\xi_{Z}+\tan \theta_{\mathrm{W}} \xi_{\gamma}=\xi_{W}
$$

For $\xi_{1}=\xi_{2}$, one has $\xi_{\gamma}=0$ and $\xi_{Z}=\xi_{W}$.

The interaction Lagrangian (2.7) coincides with the one obtained from bilinear $R$-parity breaking $[19,33]$ if parameters are properly matched 1 . Using the results of $[19,33]$ we then obtain the partial gravitino decay widths

$$
\begin{aligned}
\Gamma\left(\psi_{3 / 2} \rightarrow h \nu_{i}\right) & =\frac{\kappa_{i} m_{3 / 2}^{3}}{384 \pi M_{\mathrm{Pl}}^{2}} \beta_{h}^{4} \\
\Gamma\left(\psi_{3 / 2} \rightarrow \gamma \nu_{i}\right) & =\frac{\kappa_{i}\left|\xi_{\gamma i}\right|^{2} m_{Z}^{2} m_{3 / 2}^{3}}{64 \pi M_{\mathrm{Pl}}^{2}} \\
\Gamma\left(\psi_{3 / 2} \rightarrow Z \nu_{i}\right) & =\frac{\kappa_{i} m_{3 / 2}^{3}}{384 \pi M_{\mathrm{Pl}}^{2}} \beta_{Z}^{2}\left(H_{Z}+16 \frac{m_{Z}^{2} \operatorname{Re}\left(\xi_{Z i}\right)}{m_{3 / 2}} G_{Z}+6 m_{Z}^{2}\left|\xi_{Z i}\right|^{2} F_{Z}\right) \\
\Gamma\left(\psi_{3 / 2} \rightarrow W^{ \pm} e_{i}^{\mp}\right) & =\frac{\kappa_{i} m_{3 / 2}^{3}}{192 \pi M_{\mathrm{Pl}}^{2}} \beta_{Z}^{2}\left(H_{W}+16 \frac{m_{W}^{2} \operatorname{Re}\left(\xi_{W i}\right)}{m_{3 / 2}} G_{W}+6 m_{W}^{2}\left|\xi_{W i}\right|^{2} F_{W}\right)
\end{aligned}
$$

where the subscript $i$ denotes the generation index. The functions $\beta_{a}, H_{a}, G_{a}$ and $F_{a}$ $(a=h, Z, W)$ are given by $[19]$

$$
\begin{aligned}
& \beta_{a}=1-\frac{M_{a}^{2}}{m_{3 / 2}^{2}}, \\
& H_{a}=1+10 \frac{M_{a}^{2}}{m_{3 / 2}^{2}}+\frac{M_{a}^{4}}{m_{3 / 2}^{4}},
\end{aligned}
$$

\footnotetext{
1 The relations are $\kappa_{i}=\left\langle\widetilde{\nu}_{i}\right\rangle / v, U_{\widetilde{Z} \widetilde{Z}}=-m_{Z} \xi_{Z}, U_{\widetilde{W} \widetilde{W}}=-m_{W} \xi_{W}, U_{\widetilde{\gamma} \widetilde{Z}}=-m_{Z} \xi_{\gamma}, U_{\widetilde{H}_{u} \widetilde{Z}} \sin \beta+$ $U_{\widetilde{H}_{d}} \widetilde{Z} \cos \beta+m_{\widetilde{\nu}_{\tau}}^{2} /\left(m_{\widetilde{\nu}_{\tau}}^{2}-m_{h}^{2}\right)=1$.
} 


$$
\begin{aligned}
G_{a} & =1+\frac{1}{2} \frac{M_{a}^{2}}{m_{3 / 2}^{2}}, \\
F_{a} & =1+\frac{2}{3} \frac{M_{a}^{2}}{m_{3 / 2}^{2}}+\frac{1}{3} \frac{M_{a}^{4}}{m_{3 / 2}^{4}} .
\end{aligned}
$$

As expected, one has for $m_{a} \xi_{a} \sim m_{a} / m_{3 / 2} \ll 1$,

$$
\Gamma\left(\psi_{3 / 2} \rightarrow h \nu_{i}\right) \simeq \Gamma\left(\psi_{3 / 2} \rightarrow Z \nu_{i}\right) \simeq \frac{1}{2} \Gamma\left(\psi_{3 / 2} \rightarrow W^{ \pm} e_{i}^{\mp}\right) .
$$

The decay width $\Gamma\left(\psi_{3 / 2} \rightarrow \gamma \nu\right)$ is of particular interest since it determines the strength of the gamma line at the end of the continuous spectrum. As discussed above, this decay width is model-dependent. Contrary to the continuous part of the spectrum it can vanish, which is the case for $\xi_{1}-\xi_{2}=0$. Generically, without such a cancellation, one obtains for the branching ratio using Eqs. (2.8) and (2.10) - (2.13):

$$
\operatorname{BR}\left(\psi_{3 / 2} \rightarrow \gamma \nu\right) \sim 0.3 \sin ^{2} \theta_{W}\left(\frac{m_{Z}}{m_{3 / 2}}\right)^{2} \sim 0.02\left(\frac{200 \mathrm{GeV}}{m_{3 / 2}}\right)^{2}
$$

This estimate will be used in Section 4 where the gamma-ray spectrum is discussed.

Another phenomenologically important issue is the flavour structure of gravitino decays, i.e., the dependence of the parameters $\xi_{\gamma i}, \xi_{Z i}$ and $\xi_{W i}$ on the generation index. In models of bilinear $R$-parity breaking, for instance, this information is encoded in the mixing parameters $\mu_{i}$,

$$
\Delta \mathcal{L}=\mu_{i} H_{u} L_{i}
$$

where $H_{u}$ and $L_{i}$ are Higgs and lepton-doublet superfields, respectively. In models of flavour the ratios of the parameters $\mu_{i}$ are related to the structure of the Majorana neutrino mass matrix,

$$
M_{\nu}=c_{i j}\left(l_{i} \phi\right)\left(l_{j} \phi\right) .
$$

An interesting example, which can account for the large mixing angles in the neutrino sector, is 'anarchy' [34] where $c_{i j}=\mathcal{O}(1)$. In this case one also has

$$
\frac{\mu_{i}}{\mu_{j}}=\mathcal{O}(1)
$$

For the purpose of illustration we shall use in the following sections the 'democratic' case $\mu_{1}=\mu_{2}=\mu_{3}$ where the parameters $\xi_{\gamma i}, \xi_{Z i}$ and $\xi_{W i}$ have no flavour dependence. Alternatively, one may consider 'semi-anarchy' (cf. [7]). Other examples can be found in [35].

In the following we shall consider gravitino masses between $100 \mathrm{GeV}$ and $600 \mathrm{GeV}$, for which the assumed hierarchy $m_{\text {SM }}^{2} \ll m_{3 / 2}^{2} \ll m_{\text {soft }}^{2}$ can only be a rough approximation. A more detailed treatment would have to incorporate mixings with heavy particles of the supersymmetric standard model. However, we find the operator analysis useful to illustrate the main qualitative features of gravitino decays. 


\section{Antimatter from gravitino decays}

The scenario of decaying gravitino dark matter provides, for a wide range of gravitino masses and lifetimes, a consistent thermal history of the Universe, incorporating successful primordial nucleosynthesis and successful baryogenesis through leptogenesis. Furthermore, if dark matter gravitinos decay at a sufficiently large rate, the decay products could be detected through an anomalous contribution to the high-energy cosmic ray fluxes. In this section we shall discuss the constraints on the gravitino parameters which follow from the observations of the positron fraction by HEAT and PAMELA and of the antiproton flux by BESS, IMAX and WiZard/CAPRICE.

The rate of antimatter production per unit energy and unit volume at the position $\vec{r}$ with respect to the center of the Milky Way is given by

$$
Q(E, \vec{r})=\frac{\rho(\vec{r})}{m_{3 / 2} \tau_{3 / 2}} \frac{d N}{d E},
$$

where $d N / d E$ is the energy spectrum of antiparticles produced in the decay, which we calculated employing event generator PYTHIA 6.4 [36]. On the other hand, $\rho(\vec{r})$ is the density profile of gravitinos in the Milky Way halo. For definiteness we shall adopt the spherically symmetric Navarro-Frenk-White halo density profile [37]:

$$
\rho(r)=\frac{\rho_{0}}{\left(r / r_{c}\right)\left[1+\left(r / r_{c}\right)\right]^{2}},
$$

with $\rho_{0} \simeq 0.26 \mathrm{GeV} / \mathrm{cm}^{3}$ and $r_{c} \simeq 20 \mathrm{kpc}$, although our conclusions are not very sensitive to the choice of the density profile.

After being produced in the Milky Way halo, charged cosmic rays propagate in the Galaxy and its vicinity in a rather complicated way before reaching the Earth. Antimatter propagation in the Milky Way is commonly described by a stationary two-zone diffusion model with cylindrical boundary conditions [38]. Under this approximation, the number density of antiparticles per unit kinetic energy, $f(T, \vec{r}, t)$, satisfies the following transport equation, which applies for both positrons and antiprotons:

$$
0=\frac{\partial f}{\partial t}=\nabla \cdot[K(T, \vec{r}) \nabla f]+\frac{\partial}{\partial T}[b(T, \vec{r}) f]-\nabla \cdot\left[\vec{V}_{c}(\vec{r}) f\right]-2 h \delta(z) \Gamma_{\text {ann }} f+Q(T, \vec{r}),
$$

where reacceleration effects and non-annihilating interactions of antimatter in the Galactic disk have been neglected, since the primary particles produced by the gravitino decay rarely cross the disk before reaching the Earth.

The first term on the right-hand side of the transport equation is the diffusion term, which accounts for the propagation through the tangled Galactic magnetic field. The

diffusion coefficient $K(T, \vec{r})$ is assumed to be constant throughout the diffusion zone and is parametrised by:

$$
K(T)=K_{0} \beta \mathcal{R}^{\delta},
$$


where $\beta=v / c$ and $\mathcal{R}$ is the rigidity of the particle, which is defined as the momentum in $\mathrm{GeV}$ per unit charge, $\mathcal{R} \equiv p(\mathrm{GeV}) / Z$. The normalization $K_{0}$ and the spectral index $\delta$ of the diffusion coefficient are related to the properties of the interstellar medium and can be determined from the flux measurements of other cosmic ray species, mainly from the Boron-to-Carbon $(\mathrm{B} / \mathrm{C})$ ratio [39]. The second term accounts for energy losses due to inverse Compton scattering on starlight or the cosmic microwave background, synchrotron radiation and ionization. The third term is the convection term, which accounts for the drift of charged particles away from the disk induced by the Milky Way's Galactic wind. It has axial direction and is also assumed to be constant inside the diffusion region: $\vec{V}_{c}(\vec{r})=$ $V_{c} \operatorname{sign}(z) \vec{k}$. The fourth term accounts for antimatter annihilation with rate $\Gamma_{\text {ann }}$, when it interacts with ordinary matter in the Galactic disk, which is assumed to be an infinitely thin disk with half-width $h=100$ pc. Lastly, $Q(T, \vec{r})$ is the source term of positrons or antiprotons, defined in Eq. (3.1). The boundary conditions for the transport equation, Eq. (3.3), require the solution $f(T, \vec{r}, t)$ to vanish at the boundary of the diffusion zone, which is approximated by a cylinder with half-height $L=1-15 \mathrm{kpc}$ and radius $R=$ $20 \mathrm{kpc}$. A detailed study shows that there is some degeneracy among the parameters of the propagation model. This can be used to decrease the size of $L$ and thereby reduce the antiproton flux from gravitino decays relative to the flux from spallation [40].

The solution of the transport equation at the Solar System, $r=r_{\odot}, z=0$, can be formally expressed by the convolution

$$
f(T)=\frac{1}{m_{3 / 2} \tau_{3 / 2}} \int_{0}^{T \max } d T^{\prime} G\left(T, T^{\prime}\right) \frac{d N\left(T^{\prime}\right)}{d T^{\prime}},
$$

where $T_{\max }=m_{3 / 2}$ for the case of the positrons and $T_{\max }=m_{3 / 2}-m_{p}$ for the antiprotons. The Green's function $G\left(T, T^{\prime}\right)$ encodes all the information about astrophysics (such as the details of the halo profile and the propagation of antiparticles in the Galaxy), while the remaining part depends on gravitino properties. Analytical and numerical expressions for the Green's function for the case of positrons and antiprotons can be found in [16].

Finally, the flux of primary antiparticles at the Solar System from gravitino decay is given by:

$$
\Phi^{\mathrm{DM}}(T)=\frac{v}{4 \pi} f(T),
$$

where $v$ is the velocity of the antimatter particle.

The calculation of the high-energy cosmic ray fluxes from gravitino decay is hindered by a large number of uncertainties stemming both from astrophysics, encoded in the Green's function, and from particle physics. However, as we shall show below, present observations of the positron fraction and the antiproton flux constrain the parameters of the model well enough to make definite predictions on the diffuse gamma-ray flux. These predictions will be tested by the Fermi LAT results in the near future, thus providing a crucial test of the scenario of decaying gravitino dark matter.

¿From the particle physics point of view, the solution to the transport equation depends on the following unknown quantities: the energy spectrum of positrons or antiprotons 
produced in the decay, the gravitino mass and the gravitino lifetime. The energy spectrum of positrons and antiprotons depends crucially on the $R$-parity breaking interactions of the gravitino. It was shown in Section 2 that when the gravitino mass is large, the branching ratios for the dominant decay channels are predicted to be $\sum_{i} \mathrm{BR}\left(\psi_{3 / 2} \rightarrow h \nu_{i}\right) \simeq 1 / 4$, $\sum_{i} \operatorname{BR}\left(\psi_{3 / 2} \rightarrow Z \nu_{i}\right) \simeq 1 / 4, \sum_{i} \operatorname{BR}\left(\psi_{3 / 2} \rightarrow W^{ \pm} e_{i}^{\mp}\right) \simeq 1 / 2$, while the branching ratio for $\psi_{3 / 2} \rightarrow \gamma \nu$ is predicted to be much smaller. Therefore, in this limit the injection spectrum of antiprotons from gravitino decay is fairly model-independent, being just a function of the gravitino mass. This is not the case, however, for the energy spectrum of positrons, since the flavour composition of the final state depends on the flavour structure of the $R$-parity breaking couplings, which cannot be predicted without invoking a model of flavour.

The observation by PAMELA of an excess in the positron fraction at energies extending at least until $100 \mathrm{GeV}$ implies a lower bound on the gravitino mass of $\sim 200 \mathrm{GeV}$ if this positron excess is interpreted in terms of gravitino decays. Besides, as shown in [10], there exists a theoretical upper bound on the gravitino mass of $\sim 600 \mathrm{GeV}$ in supergravity models with universal gaugino masses at the GUT scale, stemming from the requirement of successful thermal leptogenesis, yielding a relatively narrow range for the gravitino mass, $m_{3 / 2} \simeq 200-600 \mathrm{GeV}$. If the positron excess observed by PAMELA is unrelated to gravitino decays, the gravitino mass may be as low as $\sim 5 \mathrm{GeV}$ without yielding overclosure of the Universe [7].

\subsection{Constraints from the antiproton flux}

The interstellar antiproton flux from gravitino decay can be calculated from Eq. (3.5) using the corresponding Green's function. However, this is not the antiproton flux measured by antiproton experiments, which is affected at low energies by solar modulation. Under the force field approximation [41], the antiproton flux at the top of the Earth's atmosphere is related to the interstellar antiproton flux [42] by the simple relation

$$
\Phi_{\bar{p}}^{\mathrm{TOA}}\left(T_{\mathrm{TOA}}\right)=\left(\frac{2 m_{p} T_{\mathrm{TOA}}+T_{\mathrm{TOA}}^{2}}{2 m_{p} T_{\mathrm{IS}}+T_{\mathrm{IS}}^{2}}\right) \Phi_{\bar{p}}^{\mathrm{IS}}\left(T_{\mathrm{IS}}\right),
$$

where $T_{\mathrm{IS}}=T_{\mathrm{TOA}}+\phi_{F}$, with $T_{\mathrm{IS}}$ and $T_{\mathrm{TOA}}$ being the antiproton kinetic energies at the heliospheric boundary and at the top of the Earth's atmosphere, respectively, and $\phi_{F}$ being the solar modulation parameter, which varies between $500 \mathrm{MV}$ and $1.3 \mathrm{GV}$ over the elevenyear solar cycle. Since experiments are usually undertaken near solar minimum activity, we shall choose $\phi_{F}=500 \mathrm{MV}$ for our numerical analysis in order to compare our predicted flux with the collected data.

As discussed in [16], the calculation of the antiproton flux from gravitino decay suffers from uncertaintes in the determination of the physical parameters in the propagation of charged cosmic rays in the diffusive halo, leading to uncertainties in the magnitude of fluxes as large as two orders of magnitude at the energies relevant for present antiproton experiments. The requirement that the total antiproton flux from gravitino decay be consistent with measurements gives a lower bound on the gravitino mass which strongly depends on the choice of the halo model. In the following we shall adopt the MED propagation model, 

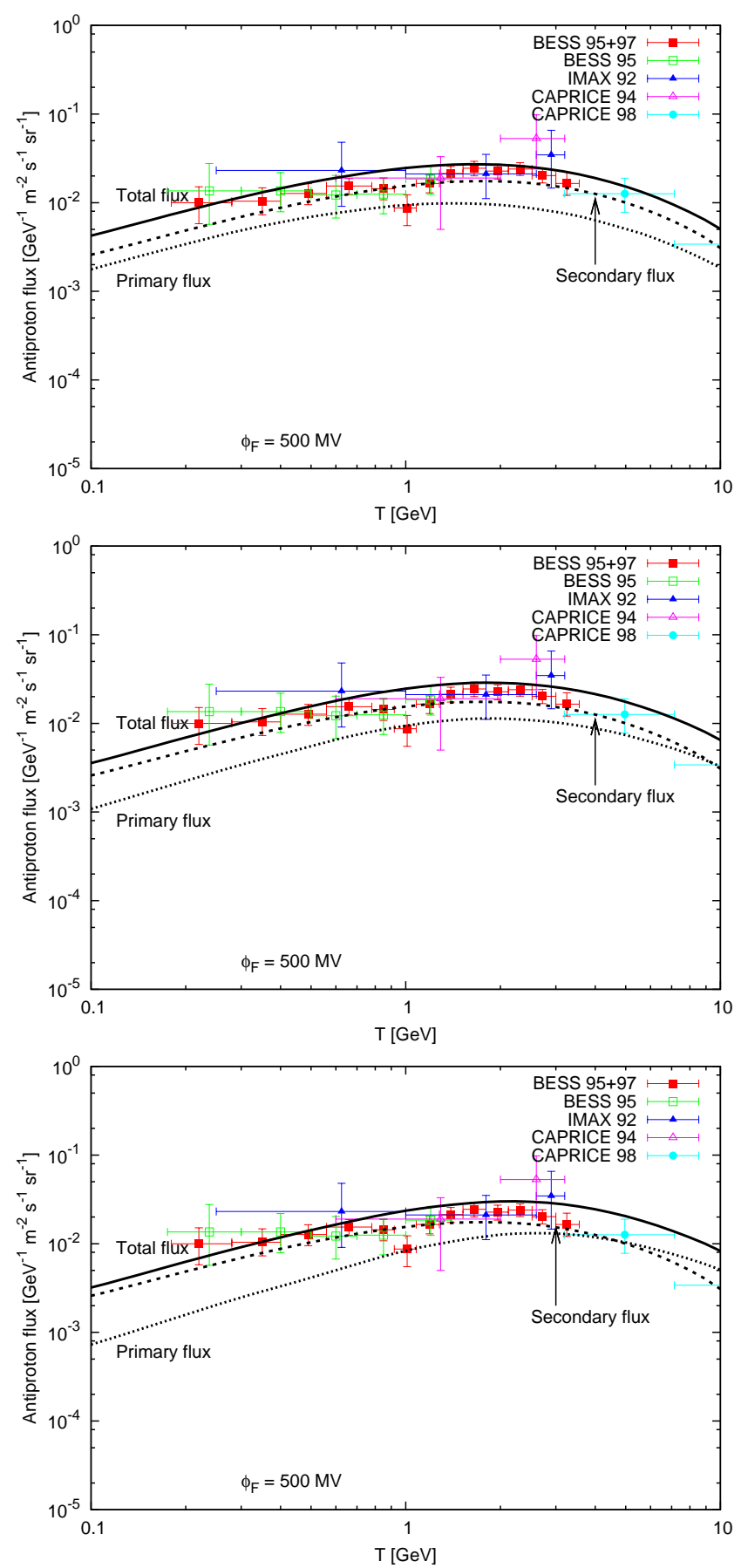

Figure 1: Antiproton fluxes for $m_{3 / 2}=200,400,600 \mathrm{GeV}$ in the MED set of propagation parameters that saturate the antiproton overproduction bound (see text). Dotted lines: antiproton flux from gravitino decays, dashed lines: secondary antiproton flux from spallation in the case of minimal nuclear cross sections, solid lines: total antiproton flux. The gravitino lifetimes are $\tau_{3 / 2}=7 \times 10^{26} \mathrm{~s}, 3 \times 10^{26} \mathrm{~s}$ and $1.5 \times 10^{26} \mathrm{~s}$, respectively. 
which provides the best fit to the $\mathrm{B} / \mathrm{C}$ ratio and measurements of flux ratios of radioactive cosmic-ray species [39].

In order to determine the maximally allowed exotic contribution to the total antiproton flux, a precise knowledge of the secondary flux of antiprotons from spallation of high-energy cosmic rays on the protons and helium nuclei in the interstellar medium is necessary. Unfortunately, the determination of this secondary flux is also subject to uncertainties. First, the choice of the propagation model can change the prediction of the secondary flux by $10-20 \%$. More importantly, the uncertainty in the nuclear cross sections for p-p, p-He, He-p and He-He collisions can change the prediction of the secondary flux by $22-25 \%$ above or below the central value [43].

A conservative upper bound on the antiproton flux from gravitinos is obtained by demanding that the total flux is not larger than the theoretical uncertainty band of the MED propagation model. This means that a 'minimal' dark matter lifetime for the MED model can be defined by a scenario where the secondary antiproton flux from spallation is $25 \%$ smaller than the central value, due to a putative overestimation of the nuclear cross sections, and the total antiproton flux saturates the upper limit of the uncertainty band which stems from astrophysical uncertainties discussed above. This amounts to the requirement that the antiproton flux from gravitino decays should not exceed $\sim 50 \%$ of the central value of secondary flux from spallation.

Using the above prescription we find the following lower bounds for the gravitino lifetime:

$$
\tau_{3 / 2}^{\min }(200) \simeq 7 \times 10^{26} \mathrm{~s}, \quad \tau_{3 / 2}^{\min }(400) \simeq 3 \times 10^{26} \mathrm{~s}, \quad \tau_{3 / 2}^{\min }(600) \simeq 1.5 \times 10^{26} \mathrm{~s},
$$

where the numbers in parentheses correspond to the gravitino masses $m_{3 / 2}=$ 200, 400 and $600 \mathrm{GeV}$, respectively. The corresponding antiproton fluxes from gravitino decay, the secondary antiproton flux from spallation and the total antiproton fluxes are shown in Figure 1 together with the experimental measurements by BESS, IMAX and WiZard/CAPRICE, and the uncertainty band from the nuclear cross sections in the MED propagation model. The minimal lifetimes (3.8) can be compared with the gravitino lifetimes needed to explain the PAMELA positron fraction excess, which will be discussed in the next section.

\subsection{Comparison with electron/positron fluxes}

Using the procedure described in the previous section it is straightforward to calculate the positron flux at Earth from gravitino decay. we shall adopt for definiteness the MED propagation model [39], characterised by $\delta=0.70, K_{0}=0.0112 \mathrm{kpc}^{2} / \mathrm{Myr}, L=4 \mathrm{kpc}$, $V_{c}=12 \mathrm{~km} / \mathrm{s}$. Note that the sensitivity of the positron fraction to the propagation model is fairly mild at the energies where the excess is observed, since these positrons were produced within a few kiloparsecs from the Earth and barely suffered the effects of diffusion.

To compare the predictions to the PAMELA results, we shall calculate the positron fraction, defined as the ratio of the positron flux to the combined positron and electron flux, $\Phi_{e^{+}} /\left(\Phi_{e^{+}}+\Phi_{e^{-}}\right)$. For the background fluxes of primary and secondary electrons, as 

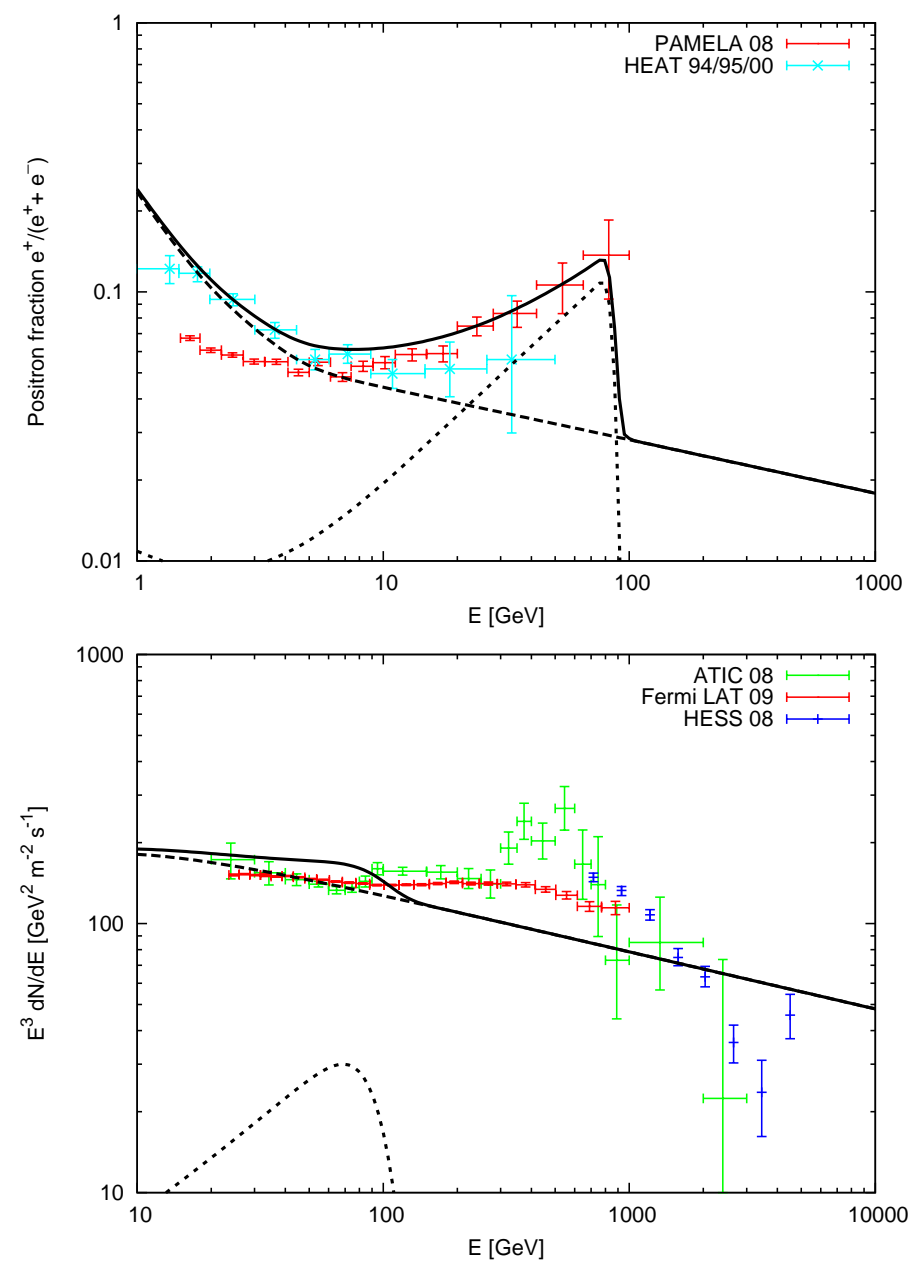

Figure 2: Contribution from dark matter decay to the positron fraction and the total electron + positron flux, compared with data from PAMELA and HEAT, and ATIC, Fermi LAT and HESS, respectively; $m_{3 / 2}=200 \mathrm{GeV}, \tau_{3 / 2}=3.2 \times 10^{26} \mathrm{~s}$, for $W^{ \pm} l^{\mp}$ decays pure electron flavour is assumed. The "Model 0" background is used, and for comparison with Fermi-LAT data $25 \%$ energy resolution is taken into account. 
well as secondary positrons, we extract the fluxes from "Model 0" presented by the Fermi collaboration in [44], which fits well the low energy data points of the total electron plus positron flux and the positron fraction, and is similar to the MED model for energies above a few $\mathrm{GeV}$ [45]. Then, the positron fraction reads

$$
\operatorname{PF}(T)=\frac{\Phi_{e^{+}}^{\mathrm{DM}}(T)+\Phi_{e^{+}}^{\mathrm{bkg}}(T)}{\Phi_{e^{+}}^{\mathrm{DM}}(T)+\Phi_{e^{+}}^{\mathrm{bkg}}(T)+\Phi_{e^{-}}^{\mathrm{DM}}(T)+k \Phi_{e^{-}}^{\mathrm{bkg}}(T)},
$$

where $k=\mathcal{O}(1)$ is the normalization of the astrophysical contribution to the primary electron flux, which is chosen to provide a qualitatively good fit to the data.

We now discuss the hypothesis that the PAMELA positron excess is due to gravitino dark matter decay. For this to be the case, the gravitino mass must be at least $200 \mathrm{GeV}$. As discussed above, the branching ratios into Standard Model particles will be essentially fixed for gravitino masses of a few hundred $\mathrm{GeV}$. The decay $\psi_{3 / 2} \rightarrow W^{ \pm} \ell^{\mp}$ then has a branching ratio of $\sim 50 \%$, and the hard leptons that are directly produced in these decays may account for the rise in the positron fraction if a significant fraction of these leptons has electron or muon flavour.

Consider first the extreme case that the decays occur purely into electron flavour. For $m_{3 / 2}=200 \mathrm{GeV}$, the PAMELA excess can then be explained for the gravitino lifetime $\tau_{3 / 2}^{e}(200) \simeq 3.2 \times 10^{26} \mathrm{~s}$, as illustrated by Figure 2. Note that this lifetime is a factor 2 smaller than the minimum lifetime given in (3.8), which we obtained from the antiproton constraint. In other words, an interpretation of the PAMELA excess in terms of gravitino decays is incompatible with the MED set of propagation parameters once antiprotons are taken into account. Nevertheless, the MIN model and other sets of parameters that yield intermediate values for the antiproton flux can easily be compatible with both the positron fraction and the antiproton-to-proton ratio observed by PAMELA. The situation is very similar for $m_{3 / 2}=400$ and $600 \mathrm{GeV}$.

Figure 2 also shows the predicted total electron + positron flux together with the results from Fermi and ATIC. Obviously, the "Model 0" presented by the Fermi collaboration in [44], cannot account for the present data, and the contribution from gravitino decays makes the discrepancy even worse. In particular, the data show no spectral feature expected for decaying dark matter. On the other hand, gravitino decays may very well be consistent with the measured total electron + positron flux once the background is appropriately adjusted. This is evident from Figure 3 where the contribution from gravitino decays is shown in the theoretically well motivated case of flavour democratic decays. The figure also illustrates that, depending on the gravitino mass, the dark matter contribution to the PAMELA excess can still be significant.

An obvious possibility is that both, the total electron + positron flux and the positron fraction, are dominated by astrophysical sources. For instance, for the gravitino mass $m_{3 / 2}=100 \mathrm{GeV}$ we obtain from the antiproton flux constraint $\tau_{3 / 2}^{\min }(100) \simeq 1 \times 10^{27} \mathrm{~s}$. As Figure 4 demonstrates, the contribution from gravitino decays to the total electron + positron flux and positron fraction is indeed negligible. Nevertheless, as we shall see in the following section, the dark matter contribution to the gamma-ray flux can be sizable. 

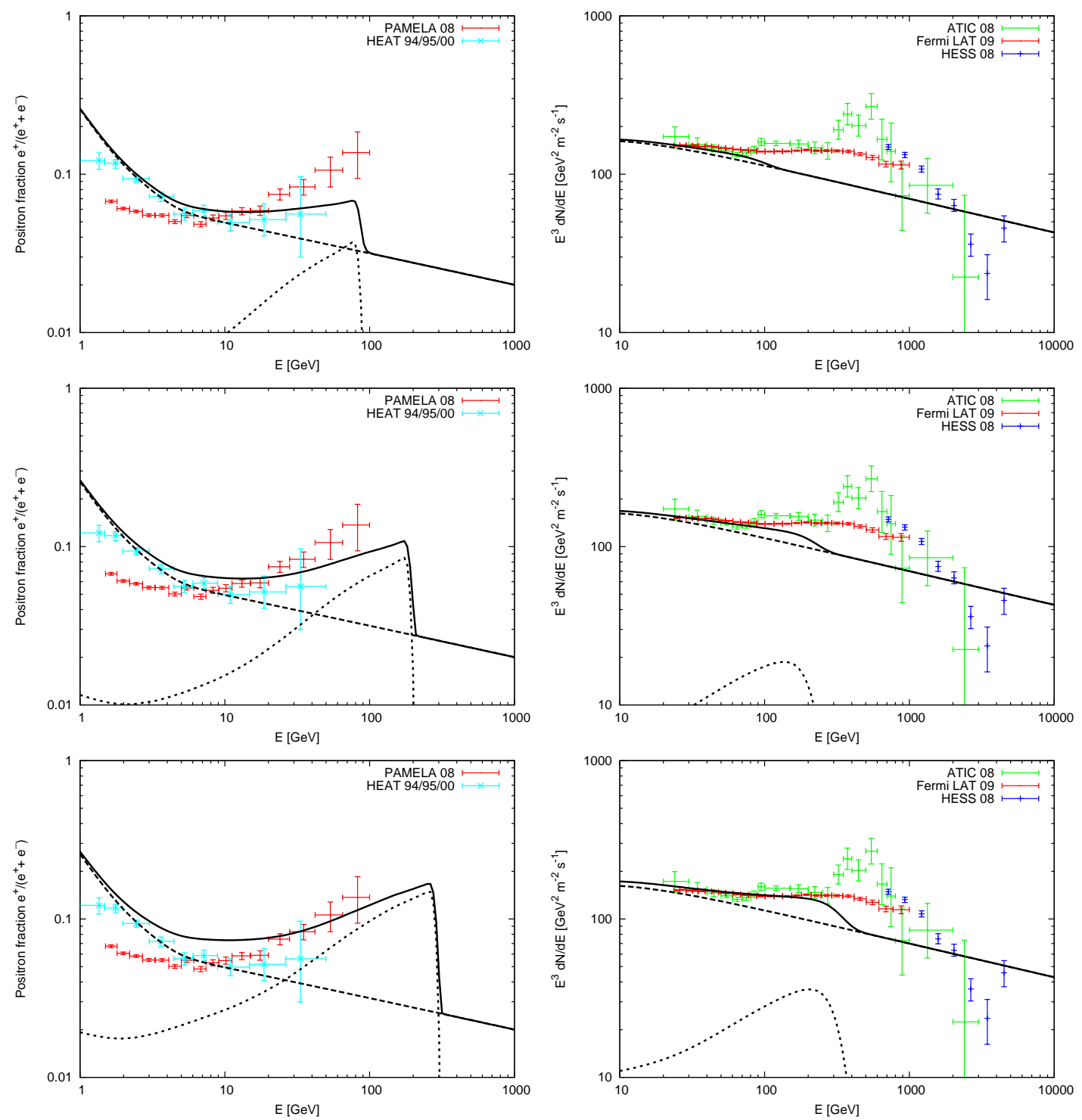

Figure 3: Contribution from dark matter decay to the positron fraction and the total electron + positron flux, compared with data from PAMELA and HEAT, and ATIC, Fermi LAT and HESS, respectively; $m_{3 / 2}=200,400,600 \mathrm{GeV}$ with the minimal lifetimes (3.8) (top to bottom); for $W^{ \pm} l^{\mp}$ decays democratic flavour dependence is assumed. The "Model 0" background is used, and for comparison with Fermi LAT data 25\% energy resolution is taken into account. 

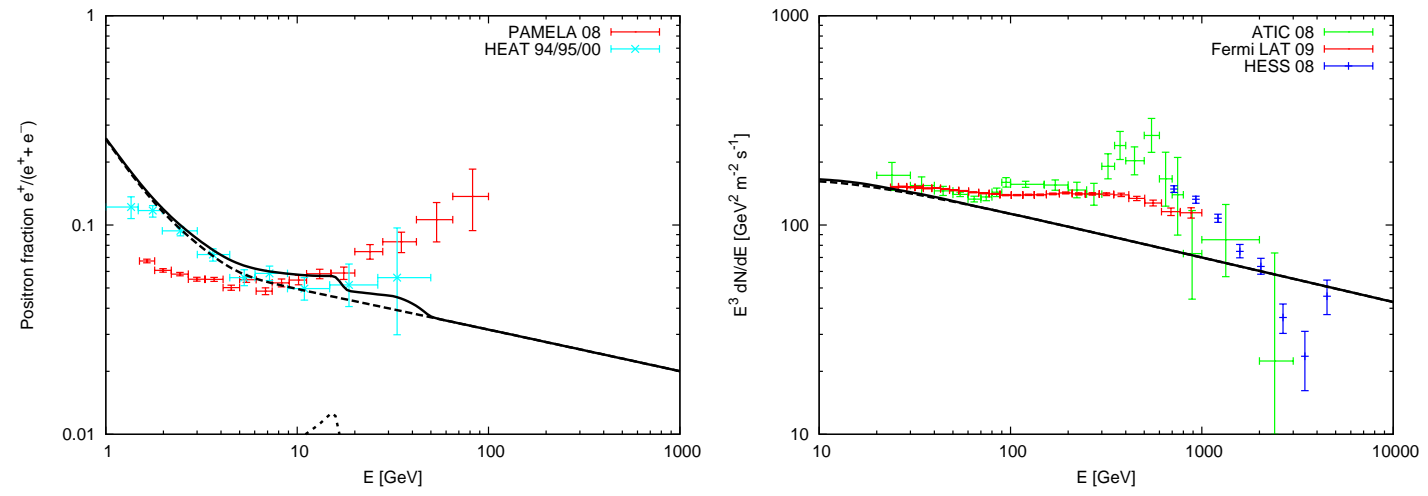

Figure 4: Contribution of gravitino decays to positron fraction and total electron + positron flux for $m_{3 / 2}=100 \mathrm{GeV}$ and $\tau_{3 / 2}=1 \times 10^{27} \mathrm{~s}$.

\section{Predictions for the diffuse gamma-ray flux}

The total gamma-ray flux from gravitino dark matter decay receives two main contributions. The first one stems from the decay of gravitinos in the Milky Way halo,

$$
\left[E^{2} \frac{d J}{d E}\right]_{\text {halo }}=\frac{2 E^{2}}{m_{3 / 2}} \frac{d N_{\gamma}}{d E} \frac{1}{8 \pi \tau_{3 / 2}} \int_{\text {los }} \rho_{\text {halo }}(\vec{l}) d \vec{l},
$$

where $d N_{\gamma} / d E$ is the gamma-ray spectrum produced in the gravitino decay. The integration extends over the line of sight, so the halo contribution has an angular dependence on the direction of observation.

In addition to the cosmological contribution, the total gamma-ray flux also receives a contribution from the decay of gravitinos at cosmological distances, giving rise to a perfectly isotropic extragalactic diffuse gamma-ray background. The flux received at the Earth with extragalactic origin is given by

$$
\left[E^{2} \frac{d J}{d E}\right]_{\mathrm{eg}}=\frac{2 E^{2}}{m_{3 / 2}} C_{\gamma} \int_{1}^{\infty} d y \frac{d N_{\gamma}}{d(E y)} \frac{y^{-3 / 2}}{\sqrt{1+\Omega_{\Lambda} / \Omega_{M} y^{-3}}},
$$

where $y=1+z, z$ being the redshift, and

$$
C_{\gamma}=\frac{\Omega_{3 / 2} \rho_{c}}{8 \pi \tau_{3 / 2} H_{0} \Omega_{M}^{1 / 2}} \simeq 10^{-6}\left(\mathrm{~cm}^{2} \mathrm{~s} \mathrm{str}\right)^{-1} \mathrm{GeV}\left(\frac{\tau_{3 / 2}}{10^{27} \mathrm{~s}}\right)^{-1} .
$$

Here, $\Omega_{3 / 2}, \Omega_{M}$ and $\Omega_{\Lambda}$ are the gravitino, matter and cosmological constant density parameters, respectively, $\rho_{c}$ is the critical density and $H_{0}$ the present value of the Hubble parameter.

The cosmological contribution is numerically smaller than the halo contribution. Moreover, the flux of gamma-rays of cosmological origin is attenuated by the electron-positron 

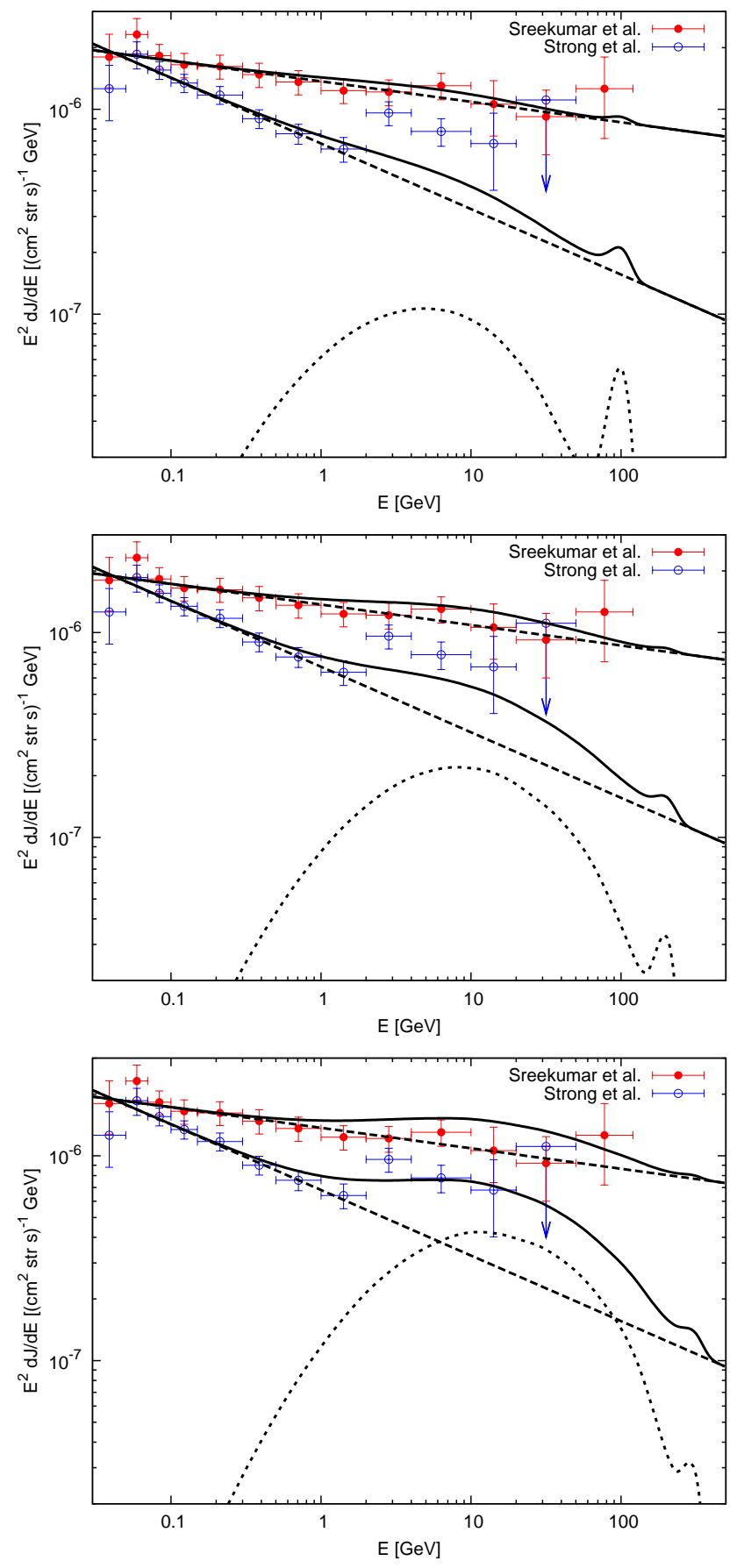

Figure 5: Predicted gamma-ray flux for $m_{3 / 2}=200,400,600 \mathrm{GeV}$ for the minimal lifetimes (3.8). We assume decays purely into electron flavour here. We show both the background obtained by Sreekumar et al. as well as the background obtained by Strong, Moskalenko and Reimer. 
pair production on the extragalactic background light emitted by galaxies in the ultraviolet, optical and infrared frequencies [46]. However, the flux of gamma-rays originating from the decay of dark matter particles in the halo is barely attenuated by pair production on the Galactic interstellar radiation field at energies below $10 \mathrm{TeV}$ [47]. Thus, the total flux is dominated by the halo component, yielding a slightly anisotropic gamma-ray flux [13] which is compatible with the EGRET observations [14]. A detailed study of the prospects of detecting this anisotropy with the Fermi LAT is beyond the scope of this paper and will be presented in more generality elsewhere [48].

The gravitino decay produces a continuous spectrum of gamma-rays which is determined by the fragmentation of the Higgs boson and the weak gauge bosons. On the other hand, there exists a gamma-ray line at the endpoint of the spectrum with an intensity which is model-dependent 2 For our numerical analysis we shall use the typical branching ratio in this channel derived in Section 2,

$$
\operatorname{BR}\left(\psi_{3 / 2} \rightarrow \nu \gamma\right)=0.02\left(\frac{200 \mathrm{GeV}}{m_{3 / 2}}\right)^{2}
$$

for gravitino masses in the range from $100-600 \mathrm{GeV}$. In Figure 5 the predicted diffuse gamma-ray flux is shown for $m_{3 / 2}=200,400,600 \mathrm{GeV}$ and the respective lower bounds (3.8) on the gravitino lifetime. These spectra correspond to upper bounds on the signal in gamma-rays that can be expected from gravitino dark matter decay.

For comparison, we show two sets of data points obtained from the EGRET measurements of the diffuse extragalactic gamma-ray background using different models of the Galactic foreground emission. An analysis by Strong, Moskalenko and Reimer using a model, optimised to better simulate the Galactic diffuse emission, revealed a power law behaviour below $1 \mathrm{GeV}$, with an intriguing deviation from a power law above $1 \mathrm{GeV}$ [17]. For our present analysis, we shall show both sets of results as the status of the extragalactic background is currently unclear. For the background obtained by Moskalenko, Strong and Reimer, the extragalactic component is described by the power law [17]

$$
\left[E^{2} \frac{d J}{d E}\right]_{\mathrm{bg}}=6.8 \times 10^{-7}\left(\frac{E}{\mathrm{GeV}}\right)^{-0.32}\left(\mathrm{~cm}^{2} \operatorname{str} \mathrm{s}\right)^{-1} \mathrm{GeV} \text {. }
$$

The earlier analysis by Sreekumar et al led to a less steep background [51],

$$
\left[E^{2} \frac{d J}{d E}\right]_{\mathrm{bg}}=1.37 \times 10^{-6}\left(\frac{E}{\mathrm{GeV}}\right)^{-0.1}\left(\mathrm{~cm}^{2} \mathrm{str} \mathrm{s}\right)^{-1} \mathrm{GeV} .
$$

\footnotetext{
${ }^{2}$ We neglect in our analysis the contribution to the gamma-ray flux from inverse Compton scattering of high energy electrons and positrons on the interstellar radiation field. We estimate that this contribution is peaked at energies smaller than $0.1 \mathrm{GeV}$ and has an intensity $E^{2} \frac{d J}{d E} \lesssim \mathcal{O}\left(10^{-7}\right)\left(\mathrm{cm}^{2} \mathrm{str} \mathrm{s}\right)^{-1} \mathrm{GeV}$ [49], thus giving a negligible contribution to the total flux, which is constrained by EGRET to be $E^{2} \frac{d J}{d E} \sim$ $10^{-6}\left(\mathrm{~cm}^{2} \operatorname{str~s}\right)^{-1} \mathrm{GeV}$ at $E=0.1 \mathrm{GeV}$. This contribution, however, can be sizable for larger dark matter masses $[49,50]$.
} 


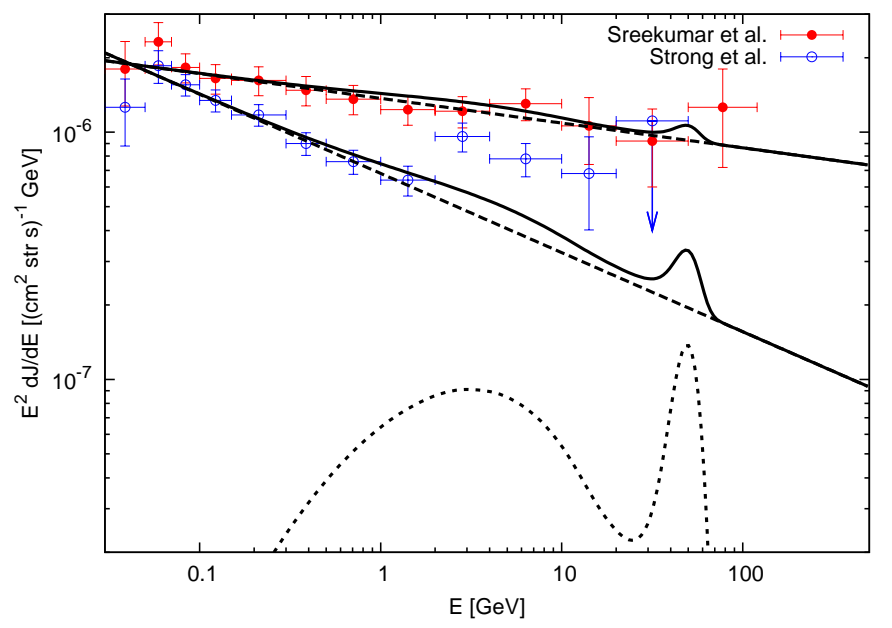

Figure 6: Predicted gamma-ray flux for $m_{3 / 2}=100 \mathrm{GeV}$ and $\tau_{3 / 2}=1 \times 10^{27} \mathrm{~s}$. We show both the background obtained by Sreekumar et al. as well as the background obtained by Strong, Moskalenko and Reimer.

For comparison with the data points, we have averaged the slightly anistropic halo signal over the whole sky, excluding a band of $\pm 10^{\circ}$ around the Galactic disk 3 We have conservatively used an energy resolution $\sigma(E) / E=15 \%$ as quoted by Fermi [52].

It is remarkable that for both choices of the extragalactic background, the antiproton constraint allows for a sizable deviation from a power law background if the gravitino mass is above $200 \mathrm{GeV}$. Therefore, if such a deviation with the proper angular dependence is observed by Fermi LAT, the scenario of gravitino dark matter will gain support. Furthermore, the existence of a gamma-ray line at the end of the spectrum is predicted, with an intensity that, as discussed in Section 2, depends on the model of $R$-parity breaking. This line could be observed by Fermi LAT in the diffuse gamma-ray background, but also by the ground-based Cherenkov telescopes MAGIC, HESS or VERITAS in galaxies such as M31 [13]. For smaller gravitino masses the gamma-ray line becomes more prominent whereas the contribution to the continuous part of the spectrum decreases. This is illustrated in Figure 6 for $m_{3 / 2}=100 \mathrm{GeV}$.

The observation of the discussed features in the diffuse gamma-ray spectrum might, if interpreted as the result of gravitino decay, open the exciting possibility of constraining the reheating temperature of the Universe. More concretely, the thermal relic abundance

3 The halo signal would have a larger degree of anisotropy if the dark matter halo is not completely uniform but presents substructures, as suggested by N-body simulations of Milky-Way-size galaxies. In the present calculation we are interested in the average flux in the whole sky excluding the Galactic disk, which depends on the total amount of dark matter in this region and not on the way it is distributed. Therefore, for our purposes it is a good approximation to neglect substructures and to assume a smooth dark matter halo profile. 
of gravitinos is given by $[10,53,54]$

$$
\Omega_{3 / 2}^{\mathrm{th}} h^{2} \simeq 0.5\left(\frac{T_{R}}{10^{10} \mathrm{GeV}}\right)\left(\frac{100 \mathrm{GeV}}{m_{3 / 2}}\right)\left(\frac{m_{\widetilde{g}}}{1 \mathrm{TeV}}\right)^{2}
$$

Therefore, imposing that the thermal abundance of gravitinos should not be larger that the total dark matter abundance, the measurement of the gravitino mass by Fermi LAT and the measurement of the gluino mass at the LHC imply the following upper bound on the reheating temperature of the Universe:

$$
T_{R} \lesssim 2 \times 10^{9} \mathrm{GeV}\left(\frac{\Omega_{3 / 2} h^{2}}{0.1}\right)\left(\frac{100 \mathrm{GeV}}{m_{3 / 2}}\right)^{-1}\left(\frac{m_{\widetilde{g}}}{1 \mathrm{TeV}}\right)^{-2}
$$

which is saturated when all the dark matter gravitinos are of thermal origin. This bound has impoprtant implications for the scenario of thermal leptogenesis, which requires $T_{R} \gtrsim 10^{9} \mathrm{GeV}$, as well as for many inflationary scenarios.

\section{Conclusions}

In supersymmetric theories with small $R$-parity breaking thermally produced gravitinos can account for the observed dark matter, consistent with leptogenesis and nucleosynthesis. Gravitino decays then contribute to antimatter cosmic rays as well as gamma-rays. We consider gravitino masses below $600 \mathrm{GeV}$, which are consistent with universal boundary conditions at the GUT scale.

Gravitino decays into Standard Model particles can be studied in a model-independent way by means of an operator analysis. For sufficiently large gravitino masses the dimension5 operator dominates. This means that the branching ratios into $h \nu, Z \nu$ and $W^{ \pm} l^{\mp}$ are fixed, except for the dependence on lepton flavour. As a consequence, the gamma-ray flux is essentially determined once the antiproton flux is known. On the contrary, the positron flux is model-dependent.

The gamma-ray line is controlled by the dimension- 6 operator. Hence, it is suppressed compared to the continuous gamma-ray spectrum. Its strength is model-dependent and decreases with increasing gravitino mass.

Electron and positron fluxes from gravitino decays, together with the standard GALPROP background, cannot account for both, the PAMELA positron fraction and the electron + positron flux measured by Fermi LAT. For gravitino dark matter, the observed fluxes require astrophysical sources. However, depending on the gravitino mass and the background, the dark matter contribution to the electron and positron fluxes can be nonnegligable.

Present data on charged cosmic rays allow for a sizable contribution of gravitino dark matter to the gamma-ray spectrum, in particular a line at an energy below $300 \mathrm{GeV}$. Non-observation of such a line would place an upper bound on the gravitino lifetime, and therefore on the strength of $R$-parity breaking, restricting possible signatures at the LHC. 


\section{Acknowledgements}

We would like to thank L. Covi, D. Horns, G. Sigl and C. Weniger for useful discussions. The work of AI and DT was partially supported by the DFG Cluster of Excellence "Origin and Structure of the Universe."

\section{References}

[1] D. Z. Freedman, P. van Nieuwenhuizen and S. Ferrara, Phys. Rev. D 13 (1976) 3214; S. Deser and B. Zumino, Phys. Lett. B 62 (1976) 335.

[2] H. Pagels and J. R. Primack, Phys. Rev. Lett. 48 (1982) 223.

[3] M. Fukugita and T. Yanagida, Phys. Lett. B 174, 45 (1986).

[4] M. Bolz, W. Buchmuller and M. Plumacher, Phys. Lett. B 443 (1998) 209. hep-ph/9809381].

[5] For a recent review and references, see

J. L. Feng, Supersymmetry and cosmology, Annals Phys. 315 (2005) 2.

[6] F. Takayama and M. Yamaguchi, Phys. Lett. B 485, 388 (2000) hep-ph/0005214].

[7] W. Buchmuller, L. Covi, K. Hamaguchi, A. Ibarra and T. Yanagida, JHEP 0703, 037 (2007) hep-ph/0702184.

[8] X. Ji, R. N. Mohapatra, S. Nussinov and Y. Zhang, Phys. Rev. D 78 (2008) 075032 [0808.1904 [hep-ph]].

[9] M. Endo and T. Shindou, 0903.1813 [hep-ph].

[10] W. Buchmuller, M. Endo and T. Shindou, JHEP 0811 (2008) 079 [0809.4667 [hep$\mathrm{ph}]]$.

[11] K. Hamaguchi, F. Takahashi and T. T. Yanagida, 0901.2168 [hep-ph].

[12] S. Lola, P. Osland and A. R. Raklev, Phys. Lett. B 656 (2007) 83 [0707.2510 [hep-ph]].

[13] G. Bertone, W. Buchmuller, L. Covi and A. Ibarra, JCAP 0711 (2007) 003 [0709.2299 [astro-ph]].

[14] A. Ibarra and D. Tran, Phys. Rev. Lett. 100, 061301 (2008) [0709.4593 [astro-ph]].

[15] K. Ishiwata, S. Matsumoto and T. Moroi, Phys. Rev. D 78 (2008) 063505 [0805.1133 [hep-ph]].

[16] A. Ibarra and D. Tran, JCAP 0807 (2008) 002 [0804.4596 [astro-ph]]. 
[17] A. W. Strong, I. V. Moskalenko and O. Reimer, Astrophys. J. 613, 956 (2004); Astrophys. J. 613 (2004) 962 astro-ph/0406254.

[18] S. W. Barwick et al. [HEAT Collaboration], Astrophys. J. 482, L191 (1997) astro-ph/9703192.

[19] L. Covi, M. Grefe, A. Ibarra and D. Tran, JCAP 0901 (2009) 029 [0809.5030 [hep-ph]].

[20] A. Ibarra and D. Tran, 0904.1410 [hep-ph].

[21] O. Adriani et al. [PAMELA Collaboration], Nature 458 (2009) 607 arXiv:0810.4995 [astro-ph]].

[22] O. Adriani et al., Phys. Rev. Lett. 102 (2009) 051101 [0810.4994 [astro-ph]].

[23] S. Orito et al. [BESS Collaboration], Phys. Rev. Lett. 84 (2000) 1078; H. Matsunaga et al., Phys. Rev. Lett. 81 (1998) 4052.

[24] J. W. Mitchell et al., Phys. Rev. Lett. 76 (1996) 3057.

[25] M. Boezio et al. [WIZARD Collaboration], Astrophys. J. 487 (1997) 415; M. Boezio et al. [WiZard/CAPRICE Collaboration], Astrophys. J. 561 (2001) 787 astro-ph/0103513.

[26] For a recent review and references, see

L. Bergstrom, Dark Matter Candidates, 0903.4849 [hep-ph].

[27] K. Ishiwata, S. Matsumoto and T. Moroi, 0811.0250 [hep-ph]; 0903.0242 [hep-ph].

[28] A. Ibarra and D. Tran, JCAP 0902 (2009) 021 [0811.1555 [hep-ph]].

[29] J. Chang et al., Nature 456 (2008) 362.

[30] F. Aharonian et al. [H.E.S.S. Collaboration], Phys. Rev. Lett. 101 (2008) 261104 [0811.3894 [astro-ph]].

[31] A. A. Abdo et al. [The Fermi LAT Collaboration], 0905.0025 [astro-ph.HE].

[32] J. Wess and J. Bagger, Supersymmetry and supergravity, Princeton, USA: Univ. Pr. (1992) 259 p; S. Weinberg, The quantum theory of fields. Vol. 3: Supersymmetry, Cambridge, UK: Univ. Pr. (2000) 419 p

[33] M. Grefe, DESY-THESIS-2008-043

[34] L. J. Hall, H. Murayama and N. Weiner, Phys. Rev. Lett. 84 (2000) 2572 hep-ph/9911341.

[35] For a review and references, see G. Altarelli and F. Feruglio, New J. Phys. 6 (2004) 106 [hep-ph/0405048. 
[36] T. Sjöstrand, S. Mrenna and P. Skands, JHEP 0605 (2006) 026 hep-ph/0603175.

[37] J. F. Navarro, C. S. Frenk and S. D. M. White, Astrophys. J. 462 (1996) 563 astro-ph/9508025.

[38] V. S. Berezinskii, S. V. Buolanov, V. A. Dogiel, V. L. Ginzburg, V. S. Ptuskin, Astrophysics of Cosmic Rays (Amsterdam: North-Holland, 1990).

[39] D. Maurin, F. Donato, R. Taillet and P. Salati, Astrophys. J. 555 (2001) 585 astro-ph/0101231.

[40] D. Maurin, R. Taillet, F. Donato, P. Salati, A. Barrau and G. Boudoul, astro-ph/0212111.

[41] L. J. Gleeson and W. I. Axford, Astrophys. J. 149 (1967) L115; Astrophys. J. 154 (1968) 1011.

[42] J. S. Perko, Astron. Astrophys. 184 (1987) 119.

[43] F. Donato, D. Maurin, P. Salati, A. Barrau, G. Boudoul and R. Taillet, Astrophys. J. 563 (2001) 172 astro-ph/0103150.

[44] D. Grasso et al. [FERMI-LAT Collaboration], 0905.0636 [astro-ph.HE].

[45] T. Delahaye, F. Donato, N. Fornengo, J. Lavalle, R. Lineros, P. Salati and R. Taillet, arXiv:0809.5268 [astro-ph].

[46] R. J. Gould and G. P. Schreder, Phys. Rev. 155 (1967) 1404; R. C. Gilmore, P. Madau, J. R. Primack, R. S. Somerville and F. Haardt, arXiv:0905.1144 [astro-ph.CO].

[47] I. V. Moskalenko, T. A. Porter and A. W. Strong, Astrophys. J. 640 (2006) L155 arXiv:astro-ph/0511149.

[48] A. Ibarra, D. Tran and C. Weniger, in preparation.

[49] K. Ishiwata, S. Matsumoto and T. Moroi, arXiv:0905.4593 [astro-ph.CO].

[50] A. Ibarra, D. Tran and C. Weniger, arXiv:0906.1571 [hep-ph].

[51] P. Sreekumar et al. [EGRET Collaboration], Astrophys. J. 494 (1998) 523 astro-ph/9709257.

[52] For the Fermi performance see e.g. http://www-glast.slac.stanford.edu/software/IS/glast_lat_performance.htm

[53] M. Bolz, A. Brandenburg and W. Buchmuller, Nucl. Phys. B 606 (2001) 518 [Erratumibid. B 790 (2008) 336] [hep-ph/0012052].

[54] J. Pradler and F. D. Steffen, Phys. Rev. D 75 (2007) 023509 hep-ph/0608344. 